\title{
How to Intervene in the Caries Process: Dentin Caries in Primary Teeth
}

\author{
Ruth M. Santamaría ${ }^{a}$ Mohamed Hassan Abudrya ${ }^{a}$ Gülsün Gül ${ }^{b}$ \\ MHD Said Mourad $^{a}$ Grace Felix Gomez ${ }^{c}$ Andrea G. Ferreira Zandona ${ }^{b}$ \\ ${ }^{a}$ Department of Preventive and Pediatric Dentistry, University of Greifswald, Greifswald, Germany; ${ }^{b}$ Department \\ of Comprehensive Care, Tufts University, Boston, MA, USA; ' Department of Community Dentistry, Case Western \\ Reserve University, Cleveland, $\mathrm{OH}$, USA
}

\section{Keywords}

Dentin caries · Primary teeth · Nonoperative caries

management $\cdot$ Selective caries removal · Filling

\begin{abstract}
For an ORCA/EFCD consensus, this review systematically assessed available evidence regarding interventions performed and materials used to manage dentin carious lesions in primary teeth. A search for systematic reviews (SRs) and randomized clinical trials (RCTs) with a follow-up of at least 12 months after intervention was performed in PubMed, LILACS, BBO, and the Cochrane Library. The risk of bias tool from the Cochrane Collaboration and the PRISMA Statement were used for assessment of the included studies. From 101 screened articles, 2 SRs and 5 RCTs, which assessed the effectiveness of interventions in terms of pulp vitality and success of restoration, and 10 SRs and 1 RCT assessing the success of restorative materials were included. For treatments involving no carious tissue removal, the Hall technique showed lower treatment failure for approximal carious lesions compared to complete caries removal (CCR) and filling. For the treatment of deep carious lesions, techniques involving selective caries removal (SCR) showed a reduction in the incidence of pulp exposure. However, the benefit of SCR over CCR in terms of pulp symptoms or restoration success/ failure was not confirmed. Regarding restorative materials,
\end{abstract}

karger@karger.com www.karger.com/cre

Karger $\stackrel{\text { ' }}{5}$

GOPEN ACCESS
(C) 2020 The Author(s)

Published by S. Karger AG, Basel

This is an Open Access article licensed under the Creative Commons Attribution-NonCommercial-4.0 International License (CC BY-NC) (http://www.karger.com/Services/OpenAccessLicense), applicable to the online version of the article only. Usage and distribution for commercial purposes requires written permission. preformed metal crowns (PMCs) used to restore multisurface lesions showed the highest success rates compared to other restorative materials (amalgam, composite resin, glass ionomer cement, and compomer), and in the long term (1248 months) these were also less likely to fail. There is limited evidence supporting the use of PMCs to restore carious lesions with single cavities. Among nonrestorative options, silver diammine fluoride was significantly more effective in arresting caries than other treatments for treating active carious lesions of different depths. Considerable heterogeneity and bias risk were observed in the included studies. Although heterogeneity observed among the studies was substantial, the trends were similar. In conclusion, less invasive caries approaches involving selective or no caries removal seem advantageous in comparison to CCR for patients presenting with vital, symptomless, carious dentin lesions in primary teeth. There is evidence in favor of PMCs for restoring multisurface carious lesions in primary molars.

(c) 2020 The Author(s)
Published by S. Karger AG, Basel

\section{Introduction}

Since caries is no longer seen as an infectious disease [Kidd, 2011], the possibilities for lesion management have evolved. For existing dentin caries in primary teeth, management includes a wide range of approaches, in- 
cluding those where carious tissue removal is not involved, such as nonrestorative cavity control (NRCC) [Gruythuysen et al., 2011; Schwendicke et al., 2016; Santamaria et al., 2018], the use of silver fluoride products (mainly silver diammine fluoride [SDF]) [Chibinski et al., 2017; Richards, 2017], and the Hall technique (HT) [Innes et al., 2015; Santamaria and Innes, 2018]. On a wider scope, management techniques involving caries removal include those in which carious tissue is selectively removed, such as the (one-step) selective caries removal (SCR), stepwise caries removal (i.e., selective dentin carious tissue removal at the first step and in a second visit selective removal to firm dentin) [Ricketts et al., 2013; Bjørndal, 2018], and the nonselective caries removal (non-SCR), involving removal of all demineralized dentin in the cavity to reach hard dentin, leaving no softened dentin. This technique is no longer recommended [Innes et al., 2016, 2018].

The decision around when to use which treatment approach should follow the modern view of carious lesion management, which emphasizes controlling/inactivating the carious process using less invasive management approaches, avoiding initiation of the cycle of restoration, maintaining as much as possible of the affected dental tissue, and preserving the tooth for as long as possible [Schwendicke et al., 2016].

The number of clinical studies and reviews assessing and comparing the effectiveness of these diverse techniques and materials has increased over the last years. Despite the current scientific evidence regarding caries management in primary teeth, there is still no agreement on the most effective approach/material in terms of clinical success to treat carious primary teeth with dentin involvement; there is even a debate whether the outcomes should be on a tooth, child, or quality of life level. The aim of this systematic review (SR), therefore, is to systematically assess the current state of knowledge regarding interventions performed and materials used to manage carious lesions with dentin involvement in primary vital teeth, diagnosed clinically or radiographically.

\section{Materials and Methods}

This review asked the following PICO questions:

1. Are minimal invasive carious lesion management approaches (caries control and minimal operative interventions) more effective in terms of absence of signs or symptoms of pulpal disease or success of restorations or lesion arrestment than the conventional therapy (non-SCR and restoration) for managing dentin carious lesions of different depths in primary teeth?
2. What is the success/failure rate of different materials used for managing asymptomatic dentin carious lesions (occlusal or approximal) in primary teeth regarding integrity of the restoration or lesion arrest?

This study followed the PRISMA Statement recommendation [Moher et al., 2009]. No further review registration was performed as the topic was provided to the authors by the joined chairs of the ORCA/EFCD consensus workshop on how to intervene in the caries process.

\section{Search Strategy}

We included meta-analyses, SRs, and in the case of no studies for these levels of evidence, also randomized clinical trials (RCTs). We excluded studies in which caries removal was assisted by chemomechanical agents and compared to complete caries removal (CCR).

We defined the search strategy based on the combination of different predefined MeSH terms of the PubMed database. For details regarding the search terms used in English, see Table 1. Citations from: MEDLINE/PubMed, Cochrane Library, and MEDLINE via Ovid up to March 2019 were retrieved. Moreover, other electronic databases such as LILACS (Latin American \& Caribbean Health Sciences Literature), and BBO (Brazilian Library in Dentistry) were also used to identify eligible papers. We included studies performed in humans and published in English and Spanish. Records from all database searches were analyzed using Mendeley software (version 1.19.3).

\section{Inclusion Criteria \\ Participants \\ Children 3-12 years of age, primary dentition.}

\section{Intervention}

PICO Question 1. Only studies that compared a minimum of one of the following treatment approaches to non-SCR/CCR were included:

- No carious tissue removal - such as NRCC (i.e., the carious lesion is opened to allow access to the lesion for brushing, allowing continuous removal of the biofilm and remineralization using fluoride products and advising a sensible diet) [Gruythuysen et al., 2011; Schwendicke et al., 2016; Santamaria et al., 2018]; caries arresting methods (e.g., SDF or other remineralization agents), and sealing techniques including those with no caries removal using filling materials or preformed metal crowns (PMCs) (the HT; where the carious lesion is separated from the oral environment and substrate by putting in a PMC, consequently slowing or stopping the caries process [Innes et al., 2015; Santamaria and Innes, 2018].

- SCR (at one visit) - that includes [Machiulskiene et al., 2020]:

- SCR to soft dentin: the excavation of carious dentin from the peripheral walls of a deep carious lesion (excavated to hard dentin), followed by selective removal of soft dentin from the pulpal wall; or

- SCR to firm/leathery dentin: the excavation to firm/leathery dentin (physically resistant to hand excavation) in the pulpal aspect of the cavity. Periphery of the cavity should be excavated to hard dentin. Stepwise caries excavation is the excavation of dentin carious tissue removal and temporary filling at the first step and in a second visit some months later, selective removal to firm dentin. CCR to reach hard dentin is no longer recommended and considered as overtreatment. 
Table 1. Electronic databases and search strategy

PubMed, $n=3,293$ records (March 1, 2019)

PICO 1 search strategy

\#1 AND \#2

\#1 (((()(((()((Dental caries) OR caries) OR carious) OR decay) OR tooth) OR teeth) OR dentin) \#1 AND \#3

OR dental) OR lesion) OR cavity) AND primary) OR deciduous) AND ((Clinical Trial[ptyp] OR \#1 AND \#4

Review[ptyp]) AND (“1946/01/01”[PDat]: “2019/02/31”[PDat]) AND Humans[Mesh])) \#1 AND \#5

\#2 ((cavity preparation) OR selective caries removal)

\#3 ((carious tissue removal) OR caries removal)

\#4 (((stepwise) OR excavation)

\#5 (((( (non-selective) OR selective) OR partial) OR incomplete) AND remov*)

\#1 AND \#6

\#1 AND \#7

\#1 AND \#8

\#6 (((minimal) OR minimum) AND invasive)

\#7 ((pit and fissure sealants))

\#1 AND \#9

\#8 ((seal*) OR filling*)

\#9 ((crown*) OR "Hall Technique")

\#10 (((“non-restorative cavity control”) OR "non-restorative caries treatment”) OR slicing)

\#11 (("silver diamine fluoride") OR "silver fluoride")

\#12 Extract*

\#13 Space maintainer

PICO 2 search strategy

\#1 AND \#2

\#1 (((()(((()((Dental caries) OR caries) OR carious) OR decay) OR tooth) OR teeth) OR dentin) \#3 AND \#4

OR dental) OR lesion) OR cavity) AND primary) OR deciduous) AND ((Clinical Trial[ptyp] OR \#3 AND \#5

Review[ptyp]) AND (“1946/01/01”[PDat]: “2019/02/31”[PDat]) AND Humans[Mesh])) \#3 AND \#6

\#2 (((()(Dental restoration) OR permanent dental restoration) OR dental filling) OR permanent \#3 AND \#7

dental filling) OR posterior restoration) OR class I) OR Class II)

\#3 (((()(((Dental restoration) OR permanent dental restoration) OR dental filling) OR permanent

dental filling $)$ OR posterior restoration $)$ OR class I) OR Class II $))$ AND $(((((()((()(($ Dental caries $)$

OR caries) OR carious) OR decay) OR tooth) OR teeth) OR dentin) OR dental) OR lesion) OR

cavity) AND primary) OR deciduous) AND ((Clinical Trial[ptyp] OR Review[ptyp]) AND

(“1946/01/01”[PDat]: “2019/02/31”[PDat]) AND Humans[Mesh]))))

\#4 ((Dental amalgam) OR amalgam)

\#5 (((composite resins) OR compomers) OR polyacid-modified composite resin)

\#6 ((glass ionomer cements) OR glass ionomer cement)

\#7 (((resin-modified glass ionomer cement) OR high viscous glass ionomer cement) OR high viscosity glass ionomer cement)

- No caries treatment or extraction.

- Comparator/control intervention (non-SCR): that is, CCR to sound enamel and clear sound dentin (hard dentin) at one visit, usually using rotary instruments followed by restoration.

PICO Question 2. All types of materials used for restoration (i.e., amalgam [AMG], glass ionomers [self-setting or light-cured], composite resin [CR], compomer [CP], all types of PMCs), and management (i.e., fluoride products and SDF) of dentin carious lesions in primary teeth, independently of the extension of the lesion and type of cavity.

- Outcome: overall success of treatment (i.e., the absence of clinical signs or symptoms of pulpal pathology (or pain), or caries arrestment, or restoration success [satisfactory appearance of restoration, needlessness for retreatment]). We also reported subjective assessment of treatment by participants (children's parents/guardians), regardless of the outcome measure or any adverse events (e.g., gingival inflammation) or safety issues (e.g., allergies) related to the interventions.

- Follow-up: at least 12 months after intervention.

\section{Critical Appraisal}

Four investigators (R.M.S., M.H.A., A.F.G.Z., and G.G.) independently performed the search and identified the articles for potential inclusion. Data from all included studies were extracted and assessed using designed data extraction forms (Tables 2, 3). A fifth investigator (G.F.G.) resolved disagreements.

Two reviewer teams (R.M.S./M.H.A./M.S.M. and A.G.F.Z./ G.G./G.F.G.) estimated the risk of bias using the guidelines outlined by the Cochrane "risk of bias" tool [Higgins et al., 2011]. Seven criteria were considered for each included study: sequence generation, allocation concealment, masking of participants and personnel, masking of outcome assessment, incomplete outcome data, selective outcome reporting, and "other bias." Each criterion was judged as "low," "high," or "unclear" risk of bias.

PRISMA (Preferred Reporting Items for Systematic Reviews and Meta-Analyses) was used for analysis of the reporting of SRs [Moher et al., 2009]. PRISMA assessment was performed in duplicate by the 2 reviewer teams. We considered all 27 included items and considered the sum of positive answers as the final score, with higher scores indicating better reporting quality. The risk of bias 


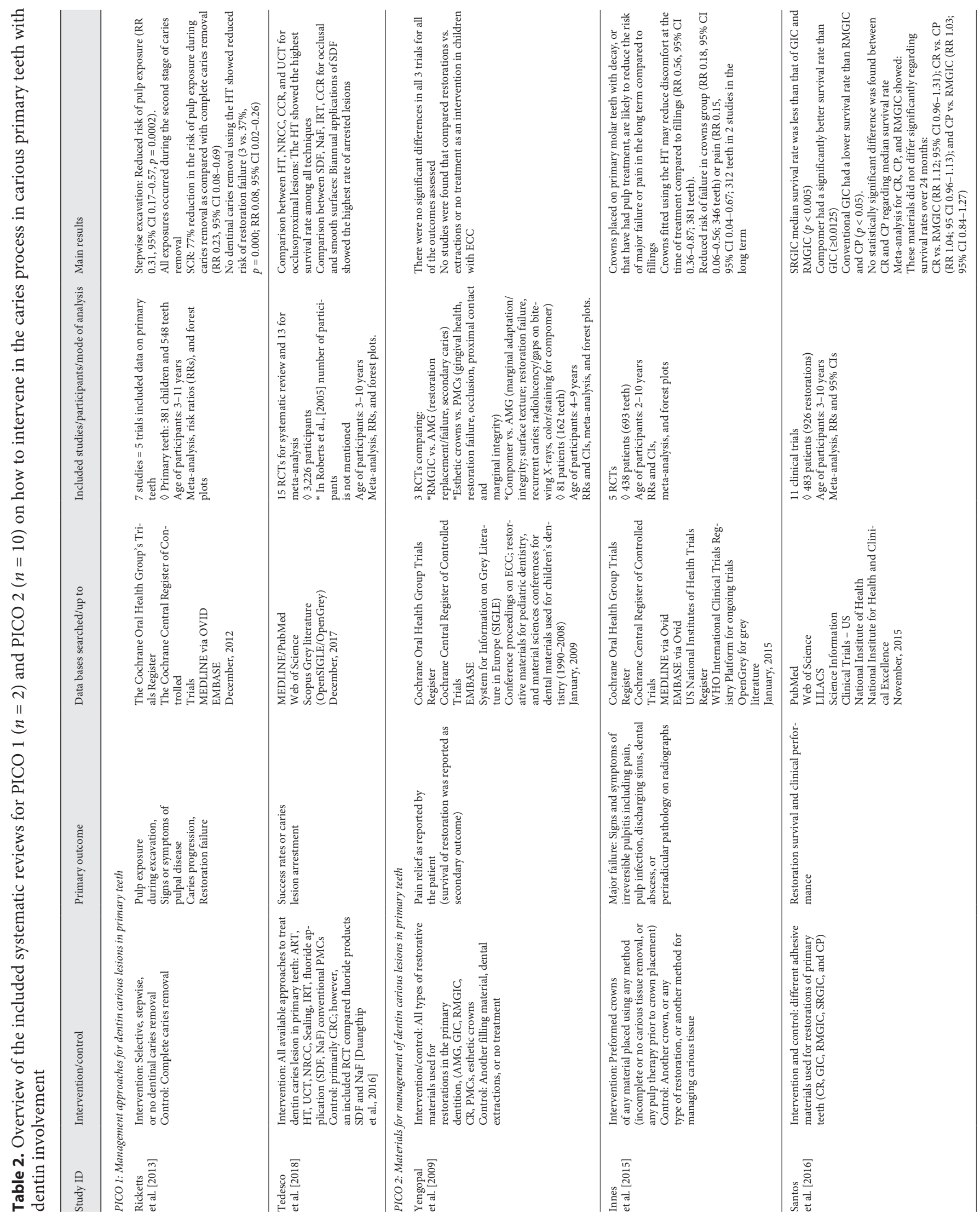




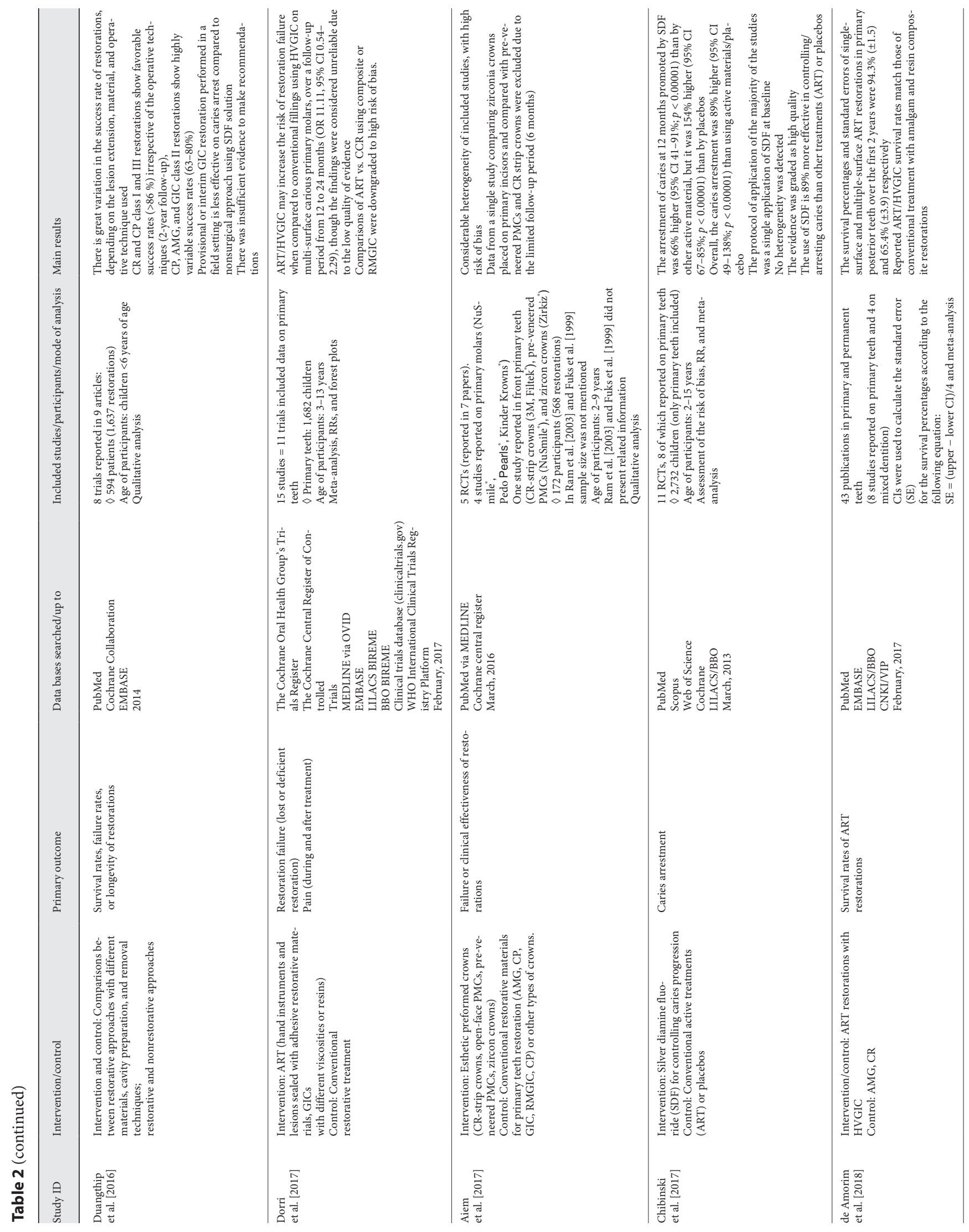




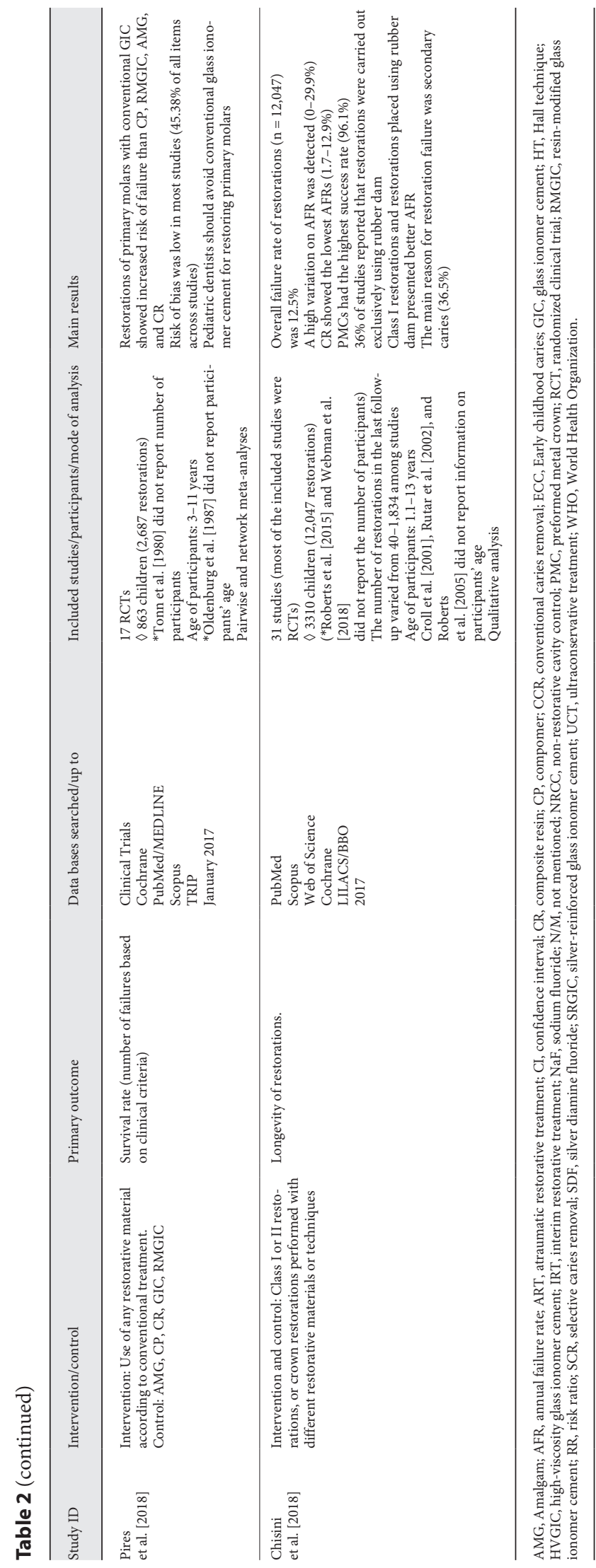

Management of Dentin Caries in Primary Teeth was interpreted as follows: $0-40 \%$ may represent a high risk of bias; $40-60 \%$ may represent a substantial risk of bias; $60-80 \%$ may represent a moderate risk of bias; $80-100 \%$ may present a low risk of bias.

\section{Assessment of Heterogeneity}

We assessed the variability across studies according to the following:

- Methodological heterogeneity: variations related to study design and risk of bias.

- Clinical heterogeneity: variations related to participants, interventions, control/comparison group(s), lesion location, lesion depth, surface(s) involved, materials used, and outcomes.

\section{Results}

\section{Selection of Studies}

The initial electronic searches in databases and other sources resulted in 4,820 records, of which 3,293 remained after duplicates were removed. After title screening, 2,673 publications were found not to be relevant. A further 519 papers were excluded after abstract screening, which left 101 publications eligible for full-text review. In total, for PICO 1 two SRs [Ricketts et al., 2013; Tedesco et al., 2018] and 5 RCTs [van Gemert-Schriks et al., 2008; Phonghanyudh et al., 2012; Mijan et al., 2014; Franzon et al., 2015; Santamaria et al., 2018], and for PICO 2 ten SRs [Yengopal et al., 2009; Innes et al., 2015; Duangthip et al., 2016; Santos et al., 2016; Aiem et al., 2017; Chibinski et al., 2017; Dorri et al., 2017; Chisini et al., 2018; de Amorim et al., 2018; Pires et al., 2018] and 1 RCT [Donly et al., 2018] met the inclusion criteria. For PICO 1, two papers [Mijan et al., 2014; Santamaria et al., 2018], which were already part of included SRs [Innes et al., 2015; Tedesco et al., 2018], were included since the SRs considered only preliminary outcomes or only some of the relevant outcomes. A flowchart of the literature search is shown in Figure 1.

\section{PICO 1: Management Approaches for Dentin Carious}

\section{Lesions in Primary Teeth}

Two SRs and meta-analysis were included: (1) Ricketts et al. [2013] assessed the effects of stepwise, selective, or no caries removal compared to complete caries removal for the treatment of dentin caries lesions in primary and permanent teeth; (2) Tedesco et al. [2018] assessed different approaches involving selective and no dentin caries removal to treat dentin carious lesions in primary teeth: atraumatic restorative treatment (ART), ultraconservative treatment (UCT; restoration of small cavities with 
ART and removal of biofilm from accessible large cavities), HT, IRT (interim restorative treatment), CCR, and so on (Table 2). Only data on primary teeth were extracted. Further details of the included SRs $(n=2)$ and RCTs $(n=5)$ are presented in Tables 2 and 3.

\section{Selective Carious Tissue Removal}

In the included studies, SCR was considered for the treatment of deep carious lesions: lesions defined as radiographically extending into the inner third or quarter of dentin, or clinically assessed at risk of pulpal exposure.

The SR by Ricketts et al. [2013] reported a significant risk reduction for pulpal exposure for one-step SCR (RR 0.23 , CI 0.08-0.69) and stepwise caries removal (RR 0.31, 95\% CI 0.17-0.57) compared to CCR for the treatment of deep carious lesions. In addition, there were no differences in pulpal symptoms between SCR and CCR (RR $0.27,95 \%$ CI $0.05-1.60, p=0.15)$ in the middle term (12 months). However, for this comparison the quality of evidence was considered low. In addition, there was insufficient evidence to determine whether there was a difference in restoration failure between SCR and CCR. For this review, it should be considered that the extension of the SCR (to soft or firm/leathery dentin) might have varied throughout the included studies. Furthermore, the exact indication of the carious tissue removal extension was not noted in the paper.

An RCT [Franzon et al., 2015] which compared the 2 -year clinical and radiographic outcomes of SCR (to leathery dentin) and CCR performed in deep carious primary molars showed no statistical benefit of SCR (66\%) over CCR $(86 \%)$ in terms of restoration survival $(p=$ 0.03 ). However, the mean incidence of pulp exposure during excavation was significantly lower in SCR (2\%) compared to CCR $(27.5 \% ; p<0.01)$. When pulp exposure during caries excavation and restoration failure - according to the modified United States Public Health Service (USPHS) Criteria [Franzon et al., 2015] - were considered together as the outcome, there was no significant difference in success rates between SCR (64\%) and CCR $(61 \% ; p=0.10)$.

A further study by Phonghanyudh et al. [2012] assessed the integrity and 1-year survival of the restoration (resin-modified glass ionomer cement [RMGIC]) for SCR (selective or complete soft dentin caries removal by hand excavation) versus CCR (rotary instruments) of lesions located in $\geq 1 / 3$ of dentin. No significant differences were reported in overall success (teeth without restoration failure, absence of pulp symptomatology, etc.) of SCR versus CCR. In terms of cavity type, the cu- mulative survival rate of Class I restorations was higher $(92-100 \%)$ than that of Class II restorations (79 and $88 \%)$.

\section{No Carious Tissue Removal}

The SR by Ricketts [2013] reported its findings based on a single study [Innes et al., 2007]. In this study asymptomatic carious lesions radiographically in $\leq$ or $>1 / 2$ way through dentin were included. Lesions were located on the occlusal (32\%) and approximal surfaces (68\%). The reported $\mathrm{dmft}$ of the study population was 2.47 . After 2 years, no dentinal caries removal using the HT showed lower failure in terms of absence of signs and symptoms of irreversible pulpitis $(2 \% ; p<0.000)$ or loss of restoration $(5 \% ; p<0.000)$ in comparison to CCR and filling (15 and $46 \%$, respectively). Restorations placed in the control group were mainly multisurface fillings restored with conventional GIC (69\%).

The SR and meta-analysis by Tedesco et al. [2018] showed that when caries arrestment is considered as the primary outcome, there are no differences in the success rates of dentin carious lesions (International Caries Detection and Assessment System [ICDAS] codes 4-5) treated with no carious tissue removal and sealed with resin materials compared to techniques involving caries removal (selective to firm/leathery dentin or CCR; RR 7.89, 95\% CI 0.39-160.91). However, for this comparison the quality of evidence was considered low due to the overall high risk of bias in the included studies. In addition, this review showed that for asymptomatic dentin carious lesions on occluso-proximal surfaces, without considering the lesion depth, the HT showed the best results in terms of restoration success, followed by the NRCC, and then treatment modalities involving CCR and conventional restoration (CP, high-viscosity GIC [HVGIC], CR, AMG, etc.).

In the RCT by Mijan et al. [2014], asymptomatic dentin carious lesions of different depths (moderate-to-deep lesions close to pulp) treated with 3 treatment modalities ART/HVGIC, UCT, and CCR/AMG were compared. In the UCT, no caries removal was performed; however, small cavities were restored with ART/HVGIC, including SCR, and medium/large cavities were left opened for daily supervised brushing. The results of this trial showed no difference in the tooth survival (absence of signs and symptoms of irreversible pulpitis) of primary molars treated according to the 3 interventions over the 3.5-year period $(90.9 \pm 2.0 \%$ with CCR, $90.4 \pm 2.4 \%$ with ART, and $88.6 \pm 1.9 \%$ with UCT; $p=0.13$ ). In addition, Mijan et al. [2014] reported that tooth survival for molars was higher for Class I cavities than 


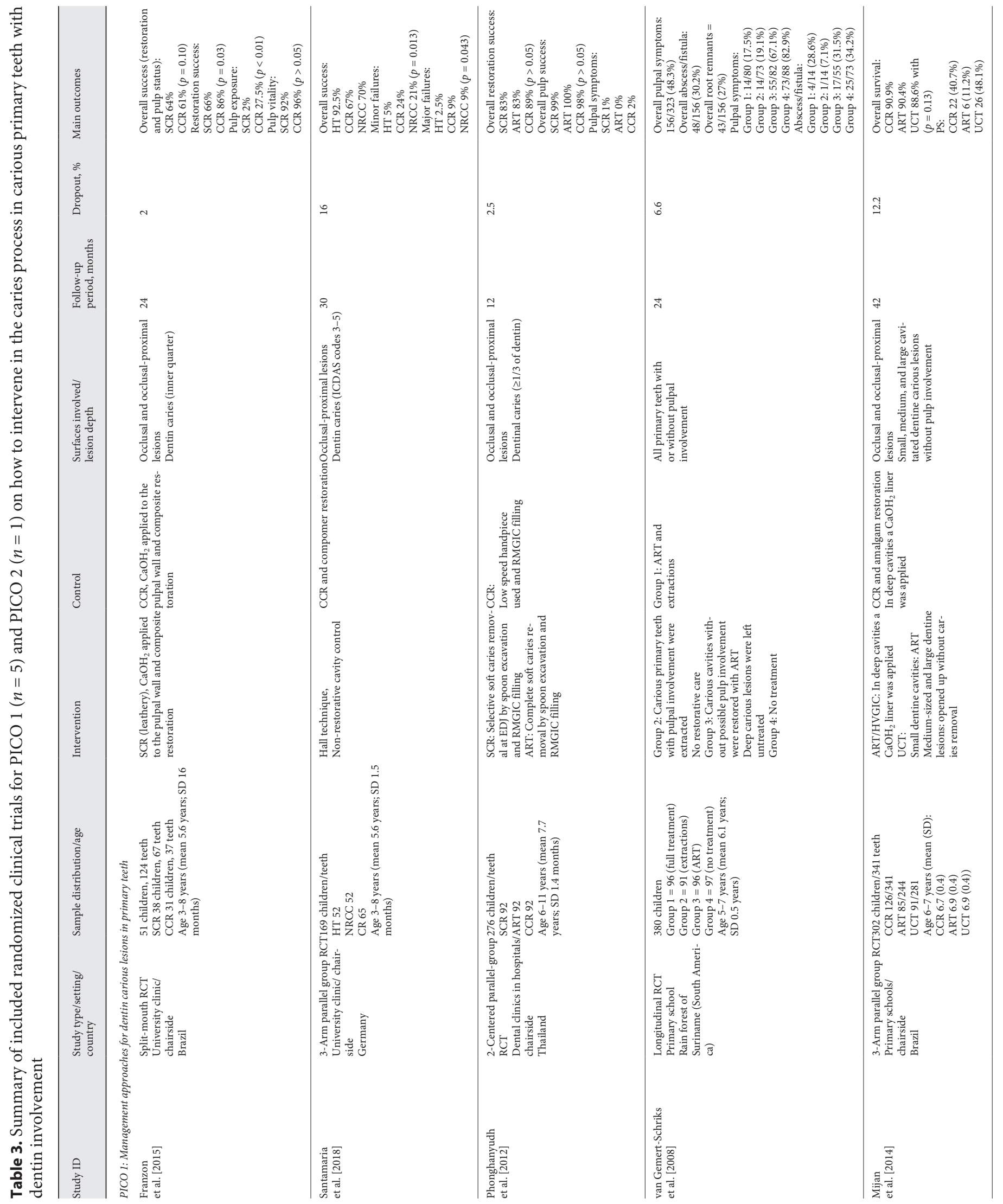




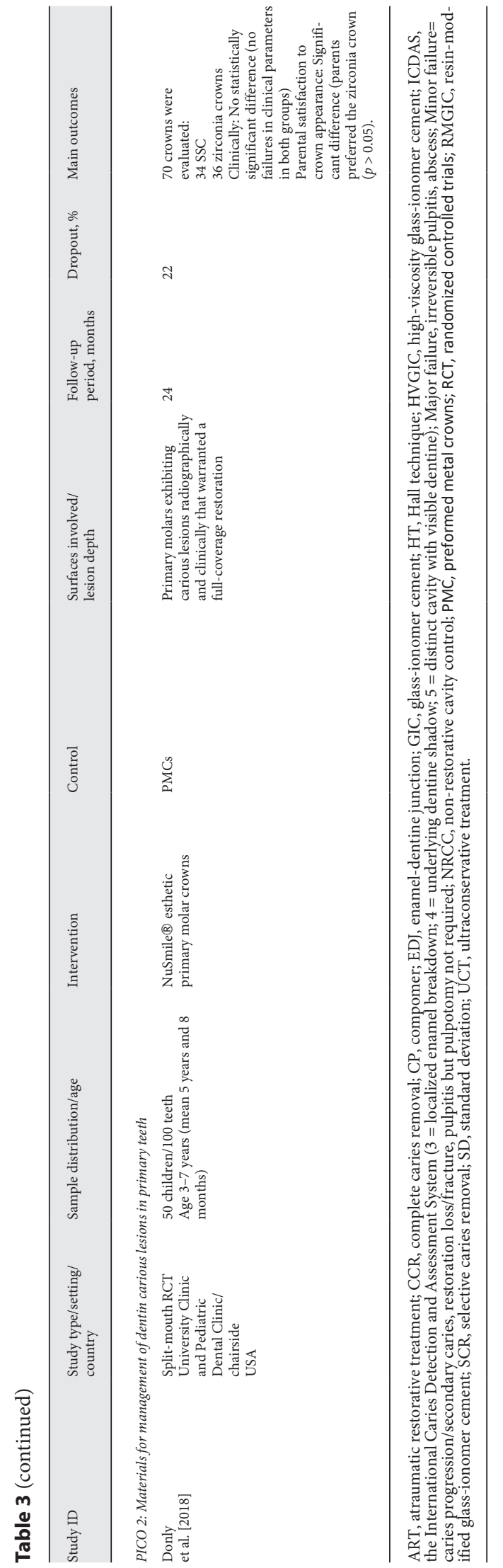

for multiple-surface cavities ( $p=0.009)$. The study was performed in a high caries risk population $(\mathrm{dmft}>5)$.

The 2.5-year report by Santamaria et al. [2018] assessed the pulp symptomatology and failure of restoration of approximal asymptomatic dentin carious lesions of different depths (ICDAS codes 3-5) treated with 2 non-caries removal techniques (NRCC and HT) vs. CCR with CP filling. Results showed significant higher success (caries arrest/restoration intact and absence of signs of pulp pathology) for teeth treated with the HT $(p=0.013)$. No significant differences were reported when NRCC and CCR with fillings were compared $(p=0.81)$. The study was performed in a high caries risk population $(\mathrm{dmft}>5)$.

No Treatment of Carious Lesions or Extraction

For no carious treatment, only 1 RCT was included [van Gemert-Schriks et al., 2008]. This study compared 4 different interventions: (1) full dental treatment (ART and extractions), (2) only extractions, (3) only restorations using ART, and (4) no treatment. No additional preventive oral health interventions were reported. After 24 months, pulpal involvement was present in $48.3 \%$ of the participants. Broken down by interventions, pulpal involvement was $17.5 \%, 19.2 \%, 67.1 \%$, and $82.9 \%$, respectively, for the 4 interventions. However, the comparison between arms in this study may have been be compromised since all teeth presenting with pulp involvement were extracted. In terms of caries increment, children treated with ART showed an increment in the $\mathrm{dmft}$ level from $5.48 \pm 3.2$ at baseline to $6.35 \pm 2.6$ after 2 years $(p<$ $0.001)$.

PICO 2: Materials for Management of Dentin Carious Lesions in Primary Teeth

Details of the included RCTs $(n=1)$ and SRs $(n=10)$ are presented in Tables 2 and 3. The included studies considered primarily restoration failure when loss, fracture, or wear of the restoration was observed and an intervention was required.

An SR and meta-analysis [Pires et al., 2018] compared the success/failure rates of different restorative materials (AMG, CR, CP, conventional GIC, RMGIC) placed in primary molars (2,687 teeth) in Class I and II cavities. The network meta-analysis showed higher risk of failure for GIC compared to CP (RR 2.64, 95\% CI 1.29-6.27), RMGIC (RR 3.25, 95\% CI 1.58-7.96), AMG (RR 2.25, 95\% CI 1.17-5.35), and CR (RR 3.27; 95\% CI 1.55-8.13). The order from lowest to highest probability of failure was RMGIC, CR, CP, AMG, and GIC. 


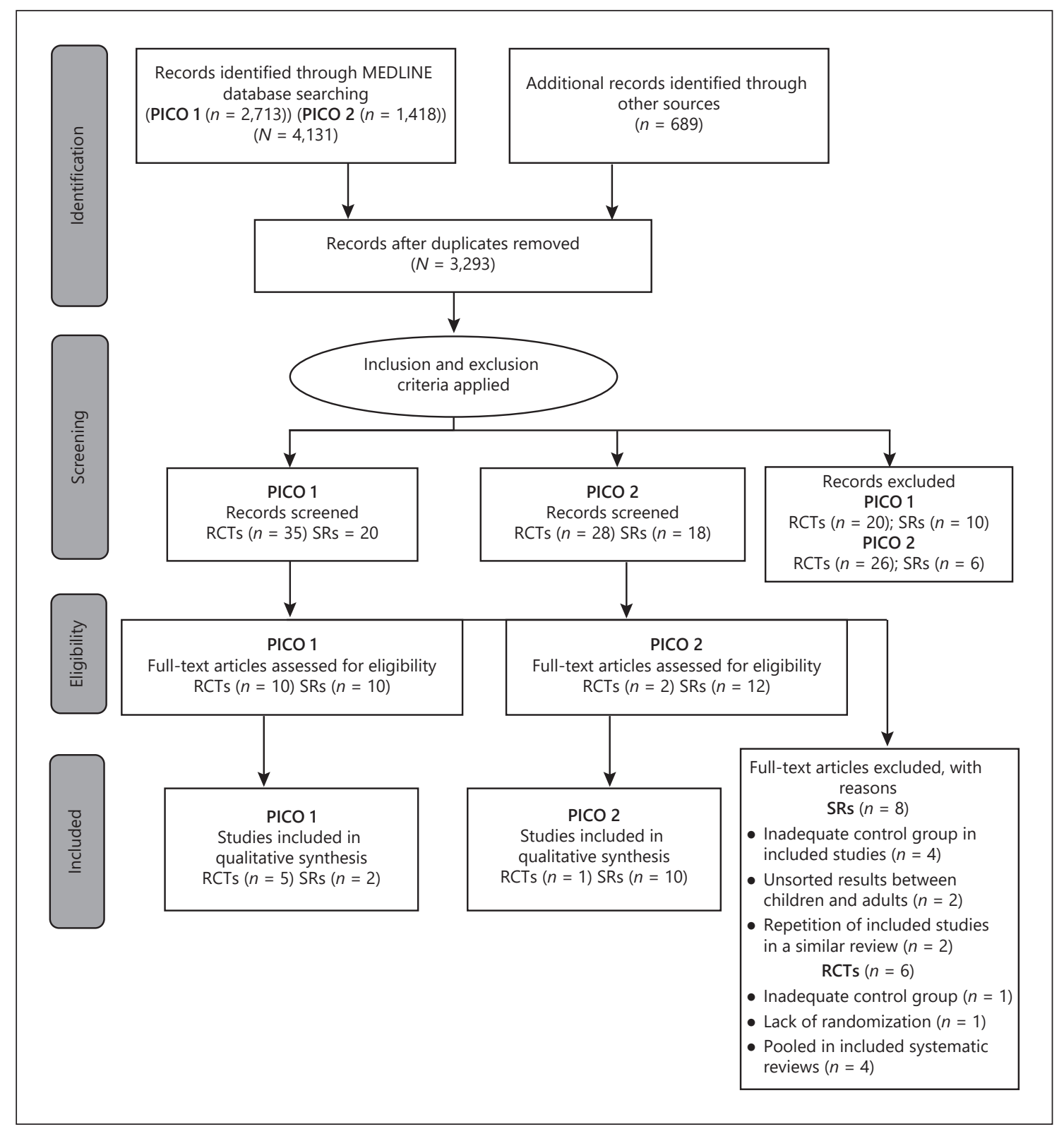

Fig. 1. Flow diagram: identification and study selection.

A further SR by Duangthip et al. [2016] assessed the effectiveness of different approaches/materials for the treatment of dentin caries in primary teeth. The subgroup analysis demonstrated that CR and CP Class I and III restorations show favorable success rates (8691\%) over a 2-year period, while CP, AMG, and GIC Class II restorations show highly variable success rates (63-80\%). Similarly, the SR by Chisini et al. [2018] reported that independently of the material, Class I restorations fail less $(7.6 \%)$ than Class II (14.7\%). In addi- tion, restorations placed under rubber dam show a better success rate than those placed without it (93.6 vs. $77.5 \%)$.

An SR and meta-analysis on ART restorations from de Amorim et al. [2018] showed for primary posterior teeth that the mean survival rates of single-surface ART/ HVGIC restorations were significantly higher (94.3 \pm $1.5 \%)$ over 2 years compared to multiple-surface ART/ HVGIC restorations $(65.4 \pm 3.9 \%)$. However, these rates were considered similar when compared to AMG resto- 
rations in primary molars. On the other hand, the SR by Dorri et al. [2017] showed that ART/HVGIC may increase the risk of restoration failure when compared to conventional fillings using composite and AMG on multisurface carious primary molars, over a follow-up period from 12 to 24 months (OR 1.11, 95\% CI 0.54-2.29). However, the findings were considered unreliable due to the low quality of evidence.

Three included SRs [Innes et al., 2015; Aiem et al., 2017; Chisini et al., 2018] and 1 RCT [Donly et al., 2018] reported on preformed crowns for restoring single and predominantly multisurface carious primary teeth. There was a wide variation in the studies related to treatment setting (chairside or general anesthesia), use of local anesthesia, number of operators, extension of the lesion surfaces involved, and so on. In general, these studies reported that in the long-term (12-48 months) PMCs were less likely to fail than conventional fillings (AMG, CR, GIC, RMGIC, and CP). Based on a single study [Innes et al., 2007], it was reported that there was no statistically significant relationship between Class I GIC restorations and the risk of a "minor" failure (restoration failure or reversible pulpitis, $p=0.272$ ), but for Class II GIC restorations the relationship was significant $(p=0.018)$. The SR by Aiem et al. [2017] also compared different types of esthetic preformed crowns (NuSmile ${ }^{\circledR}$, Pedo Pearls ${ }^{\circledR}$, and Kinder Krowns ${ }^{\circledR}$ ) for treating multisurface carious primary molars. The results of this review were inconclusive due to the overall high risk of bias with significantly dissimilar outcome measures used.

A recently published RCT [Donly et al., 2018] assessed the clinical success of zirconia crowns compared to PMCs for restoring primary molars using a split-mouth design. Fifty pairs of teeth requiring crowns were evaluated over a 2-year period. The size or extension of the carious lesions was not reported. After 2 years, 70 crowns (70\%) were assessed (zirconia crowns $=36$ [51\%], PMC $=34$ [49\%]). There were no failures in any of the groups. The authors concluded that zirconia crowns perform comparably to PMCs for restoration of primary molars.

An SR and meta-analysis by Chibinski et al. [2017] aimed to evaluate the caries-arresting effect of SDF compared to active treatments or placebos for treating active caries lesions of different depths (moderate-to-deep lesions close to pulp). The authors reported that caries arrest at 12 months using SDF was 66\% higher (RR 1.66, 95\% CI 1.41-1.96) than that by other active material (GIC restorations and fluoride varnish). In this SR, the evidence was graded as high quality.

\section{Secondary Outcomes}

The SR from Ricketts et al. [2013] reported on patient, parent/caregiver, and dentist perception outcomes during treatment when non-caries removal (HT) and CCR were compared. No dentinal caries removal using the HT was preferred by $77 \%$ of children, $83 \%$ of parents/caregivers, and $81 \%$ dentists compared to CCR and restoration. Most children (89\%) were assessed by the dentist as experiencing "no pain, discomfort" to "mild, not significant" during the intervention, compared to $78 \%$ in the CCR group.

The study from Santamaria et al. [2015] showed more child-related, negative behavior when CCR and restoration (37\%) was performed compared to NRCC (21\%) and HT (13\%). Pain intensity was rated as "low" in all treatment modalities. NRCC and HT were rated as "easy" to perform for most dentists (>77\%), compared to $50 \%$ in the non-SCR and CP filling arm. There were no significant differences in parents' rating their child's level of comfort.

Innes et al. [2015] reported that in the long term (1224 months), PMCs were less likely to cause pain than fillings. In addition, children had more discomfort with fillings than PMCs. Apparently, there is an increased risk of gingival bleeding from crowns compared to fillings in the short and long term.

Adverse events were not reported in the included studies.

Study Outcome Summary of Findings and Assessment of Heterogeneity

PICO 1

- For treatment of asymptomatic moderate-to-deep lesions, the HT involving no carious tissue removal showed lower restoration failure for multisurface cavities, when compared to CCR and restoration [Ricketts et al., 2013; Santamaria et al., 2018; Tedesco et al., 2018].

- Considering arrestment of lesions, the available evidence endorsing the similarity between sealing dentin carious lesions with resin materials without carious tissue removal and interventions involving caries tissue removal (selective to firm/leathery dentin or CCR) is limited, and low in terms of quality [Tedesco et al., 2018].

- The NRCC [in Mijan et al., 2014, presented as UCT] showed comparable results with CCR and filling for treatment of asymptomatic moderate-to-deep dentin lesions [Mijan et al., 2014; Santamaria et al., 2018]. 
However, treatment success was superior when daily toothbrushing with fluoridated toothpaste and biofilm removal was supervised. The evidence for this technique is very limited, and low in terms of quality.

- Concerning treatment of deep carious lesions (lesions extending into the inner third or quarter of dentin), SCR, one-step, and stepwise caries removal showed reduction in the incidence of pulp exposure in asymptomatic, vital, carious deciduous teeth over CCR [Ricketts et al., 2013; Franzon et al., 2015]. However, the superiority of one over the other in terms of pulp symptoms could not be confirmed.

- There is extremely limited evidence for no treatment or extraction of teeth with dentin carious lesions, and so far, these approaches cannot be recommended.

\section{PICO 2}

- There is great variation in the success rate of restorations, depending on the lesion extension, material, and operative technique used.

- Irrespective of the technique used (standard or HT), PMCs were shown to have the highest success rates compared to other filling materials and were less likely to fail than fillings [Innes et al., 2015; Aiem et al., 2017; Chisini et al., 2018].

- Considering only filling materials, probability of failure was ranked from lowest to highest: RMGIC, CR, CP, AMG, and conventional GIC [Chisini et al., 2018; Pires et al., 2018], with a single SR and meta-analysis [Santos et al., 2016] reporting no significant differences between $\mathrm{CR}, \mathrm{CP}$, and RMGIC regarding restoration survival over a 2-year period. The results of the included SRs and meta-analysis were similar. However, some clinical heterogeneity was observed among the included studies regarding caries risk of participants, isolation technique used, criteria for restoration assessment, different material brands, and so on. This could indicate some degree of bias that could compromise mainly the ranking and, therefore, the interpretation of findings.

- In general, conventional GIC showed increased failure risk than other filling materials (e.g., CP, RMGIC, AMG, and CR) [Dorri et al., 2017; Chisini et al., 2018; Pires et al., 2018].

- Considering the type of carious lesion (occlusal or approximal), there was no significant relationship between Class I GIC restorations and the risk of restoration failure or reversible pulpitis. However, a significant relationship between restoration failure and Class II GIC restorations was reported [Innes et al., 2015].

Management of Dentin Caries in Primary

Teeth
- ART/HVGIC may increase the risk of restoration failure, essentially in multisurface cavities. In contrast, ART/HVGIC was demonstrated to be an adequate management option for treating single-surface carious lesions in primary teeth [de Amorim et al., 2018]. In addition, when conventional restorations were put in comparison, ART/HVGIC survival rates showed resemblance with those of conventional treatment of AMG and resin composite restorations [de Amorim et al., 2018]. In contrast, the conflicting results by Dorri et al. [2017] could be due to the low quality of evidence, and thus were considered unreliable.

- Regardless of the material used, survival of single-surface restorations is considerably greater than that of multisurface restorations over a 2-year period [Duangthip et al., 2016; Chisini et al., 2018]. Moreover, utilization of rubber dam showed better success rates of restorations than those placed without it [Chisini et al., 2018].

- Regarding esthetic PMCs for restoring carious front teeth, there is still inconclusive evidence supporting its use over other techniques (i.e., strip crowns), primarily due to the limited follow-up ( $<12$ months). For primary molars, evidence from a single RCT [Donly et al., 2018], showed that zirconia crowns are comparable to PMCs for restoration of primary molars. However, the high risk of bias reported in the included SR (including 4 studies) [Aiem et al., 2017] prevents us from making recommendations on their effectiveness compared to PMCs.

- SDF was demonstrated to be significantly more effective in arresting caries than other treatments (fluoride varnish application and GIC restorations) or placebos for treating active caries lesions of different depths [Chibinski et al., 2017].

\section{Quality Assessment}

Considerable heterogeneity was observed in the included SRs and RCTs regarding to subject characteristics, depth of treated lesions, extension of cavities, restorative materials used, and outcome measures; however, the trends were similar. In addition, the databases searched and reporting of effect scores differed considerably among the SRs.

Most studies were found to have considerable risk of bias (Fig. 2; Table 4). All SRs reported quality of evidence as either poor or low-to-moderate level. Regarding the risk of bias of SRs, the estimated risk of bias according to 
Fig. 2. Risk of bias summary of included RCTs for PICO 1 and PICO 2 according to the Cochrane Collaboration tool.

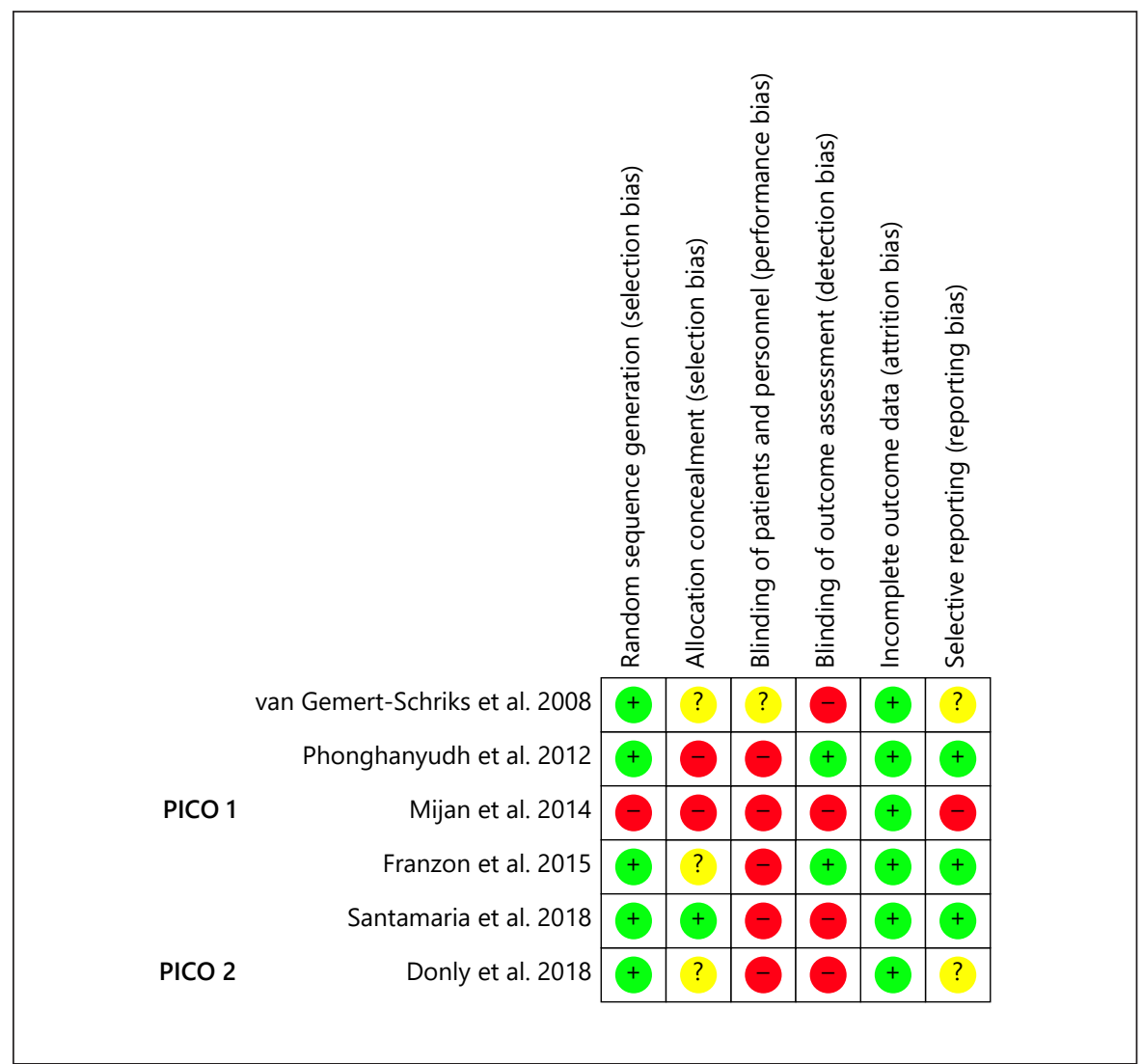

PRISMA was low for PICO 1 (100\%). For PICO 2, most of included SRs presented low risk of bias $(>80 \%)$, with 3 studies presenting a substantial (52\%) [Aiem et al., 2017] or moderate (74\%) [Duangthip et al., 2016; Chisini et al., 2018] risk of bias (Table 4).

\section{Grading the "Body of Evidence"}

Most included SRs were considered to have a low potential risk of bias. Estimation of the risk of bias related to the reporting and methodological quality of the included SRs is presented in Table 4.

\section{Discussion}

This systematic literature review aimed to assess an appropriate intervention during the caries process of dentinal lesions in terms of treatment approach (PICO 1) and material used (PICO 2) in primary teeth. Comparison of different methods of SCR, non-SCR, and treatment ap- proaches, as well of the different materials used for restorations from the selected articles, were summarized based on their benefits and limitations to concur on appropriate approaches to treat caries in primary teeth. Eligible published studies including PICO 1 (2 SRs and 5 RCTs) and PICO 2 (10 SRs and 1 RCT) that strictly met the inclusion criteria were incorporated in the analysis. Mean age varied from 5.6 to 7.7 years within the clinical trials, and from 3 to 13 years within the SRs.

Most RCTs were identified as having considerable risk of bias. Randomization was reported for all studies, occasionally without detailed clarification. On the other hand, many studies did not report on allocation concealment and blinding of participants. In general, the most frequently downgraded domains were performance and detection bias (blinding of participants/personnel or outcome). However, blinding in some of the studies could not be assured due to the dissimilarities of compared techniques and materials used (e.g., techniques with caries removal vs. no caries removal; PMCs vs. direct restorations, etc.), and the associated procedures were specific enough to identify the allocation to a certain intervention. 


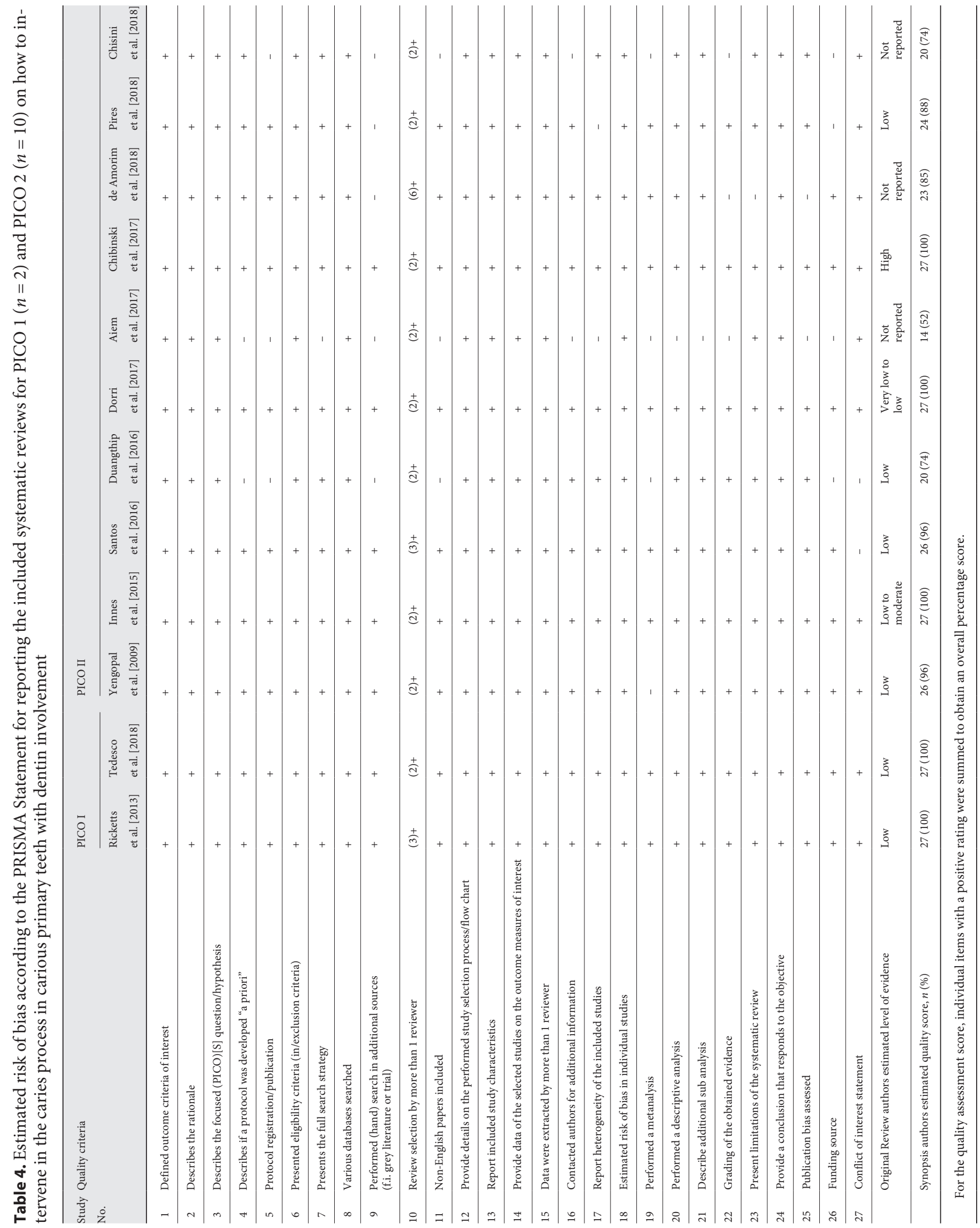


It was disappointing to have downgraded some clinical studies because these were not satisfactory in a risk of bias protocol that is essentially not applicable for this kind of studies. The protocols used for risk of bias assessment should consider and acknowledge the limitations of clinical treatment modalities. Another example of possible bias in the included studies was the variability of caries diagnostic methods and indices (dmft/DFMT, ICDAS, etc.) for treatment assessment (USPHS, own developed criteria, etc.), often without reporting calibration of examiners. The considerable risk of bias of some included studies and the heterogeneity in comparisons and outcomes (Table 3) hindered the ability to perform a metaanalysis to complete recommendations. Related to the review process, a potential risk of bias was that one of the review authors (R.M.S.) carried out one of the included studies. However, the author was not involved in the data extraction, assessment of risk of bias, or outcome analysis for this study.

Recent consensus meeting reports stated terminology and recommendations on carious tissue removal in primary and permanent teeth [Schwendicke et al., 2016; Machiulskiene et al., 2020]. In the present review, one of the causes of heterogeneity within the included studies was the depth of caries removal (i.e., the amount of tissue left or removed). Thus, what authors termed complete caries removal might not always be complete, or even SCR. Caries removal varied between studies (soft to firm dentin), generally due to the diverse and inhomogeneous use of criteria for assessing the remaining dentin tissue after carious removal. Added to this is the fact of the subjective nature of the selective removal of carious tissue and the instinctive reliance of clinicians in terms of the type of carious dentin layer that is reached. This variation might have impacted whether the pulp was exposed during caries removal. This is particularly evident in the studies which were conducted before the consensuses were published. However, our results show that particularly in the treatment of deep carious lesions in primary teeth, it seems advantageous to use techniques which involve SCR (to soft or to firm/leathery dentin) in order to reduce pulp exposure.

From the included studies there is insufficient evidence to determine whether it is necessary to re-enter as in the stepwise caries removal technique. However, an SR and meta-analysis [Schwendicke et al., 2013] (not included in present review due to pooled data reporting of primary and permanent teeth) that compared one-step incomplete and stepwise caries removal with complete caries removal showed risk reduction for pulpal exposure and pulpal symptoms for both management techniques. In the present review, the reported risk of failure for both techniques seemed to be comparable, but due to limited quality of data for this outcome, conclusions could not be drawn.

We did not address the effect of SCR on the adhesion of the restorative materials or bonding techniques, or how carious tissue removal was performed (hand, mechanical, chemomechanical, etc.). Although there is still insufficient evidence to recommend any single method/ technique for caries management, studies which involved no carious tissue removal such as the HT and sealing with resin-based materials [Ricketts et al., 2013; Tedesco et al., 2018] reported no adverse consequences when caries was left, and the lesion sealed. Findings of 2 RCTs [Borges et al., 2012; Hesse et al., 2014] included in Tedesco et al. [2018] showed a notable similarity in efficacy of sealing with resin materials regarding arrestment of asymptomatic occlusal carious lesions when compared to techniques involving carious tissue removal (SCR to firm/ leathery dentin [Hesse et al., 2014] or CCR [Borges et al., 2012]), and the possibility of avoiding CCR for dentin carious primary teeth. This may indicate that entering the carious lesion may not be necessary and that rather an accurate pulp diagnosis and adequate lesion sealing contribute to treatment success.

In addition, techniques involving no caries removal or restoration of the affected teeth (NRCC and UCT) showed similar results to complete caries removal and restoration in terms of signs or symptoms of pulp damage. Treatment success was higher when daily toothbrushing was supervised. However, data on these management approaches were of a limited quality and inconclusive. The use of NRCC is considered as an advantageous method to control carious lesion progression, to change patient/parents' behavior, and to promote oral health [Gruythuysen et al., 2011]. However, it seems to have limited clinical success, and this may limit its applicability to general situations. In general, we still need to understand the factors that support or reduce the success of NRCC, and some of these would seem to be closely related to our capacity as clinicians to change patient behavior or to aspects related to the patient and his/her family context. Further prospective long-term studies on this management technique are required, probably supplementing the caries arrestment with the use of silver fluoride agents. In the included SR [Chibinski et al., 2017], SDF showed superiority in terms of caries arrest when compared to other fluoride treatments or placebos, and the quality of this evidence was graded as high. Over- 
all, there is consistent evidence from high-quality SRs [Rosenblatt et al., 2009; Gao et al., 2016; Chibinski et al., 2017] showing the effectiveness of SDF for caries arrest in cavitated lesions in primary and permanent teeth. In general, SDF offers multiple advantages for the treatment of pediatric patients such as easy application, noninvasiveness, safety, and so on. In addition, the use of SDF buys the dentist some time until more traditional restorative methods can be utilized, if required. However, it also presents disadvantages such as the permanent black staining of the carious lesions, which can be a concern for more esthetic-oriented parents/children.

Irrespective of the technique used - standard (i.e., caries removal and tooth preparation) or HT - PMCs had the highest success rates compared to other filling materials and were less likely to fail than fillings. In general, there is evidence in favor of PMCs for the restoration of carious primary molars. Zirconia primary molar crowns show promise in terms of restoration success for the treatment of primary molars compared to PMCs [Donly et al., 2018]. However, an included review [Aiem et al., 2017] contained conflicting data based on RCTs with a high risk of bias and notably different outcomes. In addition, the cost of zirconia primary molar crowns could limit their use in daily practice, mainly considering that in many countries restorations with esthetic crowns are not covered by the statutory insurance, thus costs should be covered either by the patient or his private additional insurance. The cost-benefit of zirconia crowns for parents and health systems as compared to PMCs should also be considered in further studies. Among direct restorative materials, there is evidence against conventional GIC for the restoration of multisurface cavitated primary carious teeth as it showed increased failure risk. RMGIC, on the other hand, had the lowest failure risk followed by CR, CP, AMG, and GIC. Regarding ART using HVGICs, there is weak evidence supporting its use for the treatment of multisurface carious lesions in primary teeth. Irrespective of the material used, single-surface restorations fail less than multisurface restorations over a 2-year period [Chisini et al., 2018; Pires et al., 2018].

Whilst the technique and material per se are important for treatment success, an accurate diagnosis of the carious lesion and pulpal status are crucial and can impact the results. Authors of included studies in this review reported the inclusion of asymptomatic primary teeth with no clinical or radiographic signs of pulp damage. Thus, in daily practice for managing patients with reported pain, or other signs or symptoms of irreversible pulpitis, an- other treatment modality should be considered, which involves pulp management (pulpotomy or pulpectomy) or extraction and the use of space maintainers. In addition, patients included in most studies were individuals with high caries risk $(\mathrm{dmft}>3)$, presenting a high number of restored surfaces. This factor, although often included in clinical studies, is rarely considered as a variable of analysis and this may influence the survival of restorations. Patients' caries risk has been shown in permanent teeth to significantly influence the longevity of restorations [Demarco et al., 2012].

In general, the management of carious lesions in primary teeth is challenging. In contrast to treatment in adults, pediatric dentistry has to consider factors such as age, cognitive development, pain perception and ability to describe it, child and parents' cooperation, type of treatment, and so on. These play a central role in the selection and provision of dental treatment. To conclude, for disease control or restoration longevity, there is no single ideal therapy for managing dentin caries in pediatric patients. The current evidence shows that in symptomless, carious primary teeth, less invasive techniques involving SCR and those involving no caries removal (SDF application or the HT) could be advantageous in terms of reduction of pulp exposure or restoration failure, as compared to nonselective caries removal. In addition, for treatment of multisurface carious lesions the use of PMCs is recommendable.

\section{Further Recommendations}

Dental practitioners should consider the use of more conservative techniques involving selective or no caries removal with (HT) or without restoration (SDF) over total caries removal for patients presenting with vital, symptomless, carious lesions in primary teeth. Studies using NRCC reinforced by the use of SDF and compared to other treatment approaches are needed.

\section{Acknowledgments}

The authors wish to thank ORCA (The European Organization for Caries Research) and EFCD (The European Federation of Conservative Dentistry) for their excellent collaboration. We would like to thank the 2 reviewers of this paper, van Loveren C. (Amsterdam, The Netherlands) and Mazzoni A. (Bologna, Italy) for their valuable comments. 


\section{Statement of Ethics}

This study followed the PRISMA Statement recommendation. No further review registration was performed as the topic was provided to the authors by the joined chairs of the ORCA/EFCD consensus workshop on how to intervene in the caries process.

\section{Conflict of Interest Statement}

R.M.S., M.H.A., G.G., M.S.M., and G.F.G. declare that they have no conflict of interest. A.G.F.Z. has received research grants from Colgate, NIDCR, Greenmark, Delta Dental, and Calcivis, and is a consultant on product development by Colgate, GreenMark, and Calcivis.

\section{Author Contributions}

A.G.F.Z. and R.M.S.: designed and planned the search strategies. R.M.S., M.H.A., G.G., and A.G.F.Z.: identified studies from PubMed and screened for eligibility and inclusion. R.M.S., M.H.A, M.S.M., and A.G.F.Z.: summarized the studies and analyzed the results. R.M.S. and A.G.F.Z.: wrote a first draft of the manuscript that was complemented and revised by G.F.G., M.H.A, M.S.M, and G.G.

\section{References}

Aiem E, Smaïl-Faugeron V, Muller-Bolla M. Aesthetic preformed paediatric crowns: systematic review. Int J Paediatr Dent. 2017 Jul; 27(4):273-82.

Bjørndal L. Stepwise Excavation. Monogr Oral Sci. 2018;27:68-81.

Borges BC, De Souza Bezerra Araújo RF, Dantas RF, De Araújo Lucena A, De Assunção Pinheiro IV. Efficacy of a non-drilling approach to manage non-cavitated dentin occlusal caries in primary molars: a 12 -month randomized controlled clinical trial. Int J Paediatr Dent. 2012 Jan;22(1):44-51.

Chibinski AC, Wambier LM, Feltrin J, Loguercio AD, Wambier DS, Reis A. Silver Diamine Fluoride Has Efficacy in Controlling Caries Progression in Primary Teeth: A Systematic Review and Meta-Analysis. Caries Res. 2017; 51(5):527-41.

Chisini LA, Collares K, Cademartori MG, de Oliveira LJ, Conde MC, Demarco FF, et al. Restorations in primary teeth: a systematic review on survival and reasons for failures. Int $J$ Paediatr Dent. 2018 Mar;28(2):123-39.

de Amorim RG, Frencken JE, Raggio DP, Chen X, $\mathrm{Hu} \mathrm{X}$, Leal SC. Survival percentages of atraumatic restorative treatment (ART) restorations and sealants in posterior teeth: an updated systematic review and meta-analysis. Clin Oral Investig. 2018 Nov;22(8):2703-25.

Demarco FF, Corrêa MB, Cenci MS, Moraes RR, Opdam NJ. Longevity of posterior composite restorations: not only a matter of materials. Dent Mater. 2012 Jan;28(1):87-101.

Donly KJ, Sasa I, Contreras CI, Mendez MJ. Prospective Randomized Clinical Trial of Primary Molar Crowns: 24-Month Results. Pediatr Dent. 2018 Jul;40(4):253-8.

Dorri M, Martinez-Zapata MJ, Walsh T, Marinho VC, Sheiham Deceased A, Zaror C. Atraumatic restorative treatment versus conventional restorative treatment for managing dental caries. Cochrane Database Syst Rev. 2017 Dec;12:CD008072.
Duangthip D, Jiang M, Chu CH, Lo EC. Restorative approaches to treat dentin caries in preschool children: systematic review. Eur J Paediatr Dent. 2016 Jun;17(2):113-21.

Franzon R, Opdam NJ, Guimarães LF, Demarco FF, Casagrande L, Haas AN, et al. Randomized controlled clinical trial of the 24-months survival of composite resin restorations after one-step incomplete and complete excavation on primary teeth. J Dent. 2015 Oct;43(10): 1235-41.

Gao SS, Zhao IS, Hiraishi N, Duangthip D, Mei ML, Lo EC, et al. Clinical Trials of Silver Diamine Fluoride in Arresting Caries among Children: A Systematic Review. JDR Clin Trans Res. 2016 Oct;1(3):201-10.

Gruythuysen RJ, van Strijp AJ, van Palestein Helderman WH, Frankenmolen FW. [Non-restorative treatment of cavities in temporary dentition: effective and child-friendly]. Ned Tijdschr Geneeskd. 2011;155(42):A3489.

Hesse D, Bonifácio CC, Mendes FM, Braga MM, Imparato JC, Raggio DP. Sealing versus partial caries removal in primary molars: a randomized clinical trial. BMC Oral Health. 2014 May;14(1):58.

Higgins JP, Altman DG, Gøtzsche PC, Jüni P, Moher $\mathrm{D}$, Oxman $\mathrm{AD}$, et al.; Cochrane Bias Methods Group; Cochrane Statistical Methods Group. The Cochrane Collaboration's tool for assessing risk of bias in randomised trials. BMJ. 2011 Oct;343:d5928.

Innes N, Schwendicke F, Frencken J. An Agreed Terminology for Carious Tissue Removal. Monogr Oral Sci. 2018;27:155-61.

Innes NP, Evans DJ, Stirrups DR. The Hall Technique; a randomized controlled clinical trial of a novel method of managing carious primary molars in general dental practice: acceptability of the technique and outcomes at 23 months. BMC Oral Health. 2007 Dec;7(1): 18.
Innes NP, Frencken JE, Bjørndal L, Maltz M, Manton DJ, Ricketts D, et al. Managing Carious Lesions: Consensus Recommendations on Terminology. Adv Dent Res. 2016 May; 28(2):49-57.

Innes NP, Ricketts D, Chong LY, Keightley AJ, Lamont T, Santamaria RM. Preformed crowns for decayed primary molar teeth. Cochrane Database Syst Rev. 2015 Dec;(12):CD005512.

Kidd E. The implications of the new paradigm of dental caries. J Dent. 2011 Dec;39 Suppl 2:S38.

Machiulskiene V, Campus G, Carvalho J, Dige I, Ekstrand K, Jablonski-Momeni A, et al. Terminology of Dental Caries and Dental Caries Management: Consensus Report of a Workshop Organized by ORCA and Cariology Research Group of IADR. Caries Res. 2020; 54(1):7-14.

Mijan M, de Amorim RG, Leal SC, Mulder J, Oliveira L, Creugers NH, et al. The 3.5-year survival rates of primary molars treated according to three treatment protocols: a controlled clinical trial. Clin Oral Investig. 2014 May;18(4):1061-9.

Moher D, Liberati A, Tetzlaff J, Altman DG; PRISMA Group. Preferred reporting items for systematic reviews and meta-analyses: the PRISMA statement. PLoS Med. 2009 Jul; 6(7):e1000097.

Phonghanyudh A, Phantumvanit P, Songpaisan Y, Petersen PE. Clinical evaluation of three caries removal approaches in primary teeth: a randomised controlled trial. Community Dent Health. 2012 Jun;29(2):173-8.

Pires CW, Pedrotti D, Lenzi TL, Soares FZ, Ziegelmann PK, Rocha RO. Is there a best conventional material for restoring posterior primary teeth? A network meta-analysis. Braz Oral Res. 2018 Mar;32(0):e10.

Richards D. The effectiveness of silver diamine fluoride in arresting caries. Evid Based Dent. 2017 Oct;18(3):70. 
Ricketts D, Lamont T, Innes NP, Kidd E, Clarkson JE. Operative caries management in adults and children. Cochrane Database Syst Rev. 2013 Mar;(3):CD003808.

Rosenblatt A, Stamford TC, Niederman R. Silver diamine fluoride: a caries "silver-fluoride bullet”. J Dent Res. 2009 Feb;88(2):116-25.

Santamaría R, Innes N. Sealing Carious Tissue in Primary Teeth Using Crowns: The Hall Technique. Monogr Oral Sci. 2018;27:113-23.

Santamaria RM, Innes NP, Machiulskiene V, Evans DJ, Alkilzy M, Splieth CH. Acceptability of different caries management methods for primary molars in a RCT. Int J Paediatr Dent. 2015 Jan;25(1):9-17.
Santamaría RM, Innes NP, Machiulskiene V, Schmoeckel J, Alkilzy M, Splieth CH. Alternative Caries Management Options for Primary Molars: 2.5-Year Outcomes of a Randomised Clinical Trial. Caries Res. 2018 Jan;51(6):60514.

Santos AP, Moreira IK, Scarpelli AC, Pordeus IA, Paiva SM, Martins CC. Survival of Adhesive Restorations for Primary Molars: A Systematic Review and Metaanalysis of Clinical Trials. Pediatr Dent. 2016 Oct;38(5):370-8.

Schwendicke F, Dörfer CE, Paris S. Incomplete caries removal: a systematic review and metaanalysis. J Dent Res. 2013 Apr;92(4):306-14.

Schwendicke F, Frencken JE, Bjørndal L, Maltz M, Manton DJ, Ricketts D, et al. Managing Carious Lesions: Consensus Recommendations on Carious Tissue Removal. Adv Dent Res. 2016 May;28(2):58-67.
Tedesco TK, Gimenez T, Floriano I, Montagner AF, Camargo LB, Calvo AF, et al. Scientific evidence for the management of dentin caries lesions in pediatric dentistry: A systematic review and network meta-analysis. PLoS One. 2018 Nov; 13(11):e0206296.

van Gemert-Schriks MC, van Amerongen WE, ten Cate JM, Aartman IH. The effect of different dental treatment strategies on the oral health of children: a longitudinal randomised controlled trial. Clin Oral Investig. 2008 Dec; 12(4):361-8.

Yengopal V, Harneker SY, Patel N, Siegfried N. Dental fillings for the treatment of caries in the primary dentition. Cochrane Database Syst Rev. 2009 Apr;(2):CD004483. 